\title{
Short-term efficacy of precise hepatectomy and traditional hepatectomy for primary liver cancer: a systematic review and meta-analysis
}

\author{
Xu Niu ${ }^{1 \#}$, Jing Liu ${ }^{2 \#}$, Zhangdong Feng ${ }^{1}$, Teng Zhang ${ }^{1}$, Tuo Su ${ }^{1}$, Wei Han ${ }^{1}$ \\ ${ }^{1}$ Department of General Surgery, Beijing Luhe Hospital Affiliated to Capital Medical University, Beijing, China; ${ }^{2}$ Department of General Surgery, \\ Beijing Tongren Hospital, Capital Medical University, Beijing, China \\ Contributions: (I) Conception and design: X Niu; (II) Administrative support: J Liu; (III) Provision of study materials or patients: Z Feng; (IV) \\ Collection and assembly of data: T Zhang; (V) Data analysis and interpretation: T Su, W Han; (VI) Manuscript writing: All authors; (VII) Final \\ approval of manuscript: All authors. \\ \#These authors contributed equally to this work. \\ Correspondence to: Wei Han. Department of General Surgery, Beijing Luhe Hospital Affiliated to Capital Medical University, Beijing 11149 , China. \\ Email: grnathan1@163.com.
}

Background: As the base of hepatitis B patients has been increasing annually, it has developed into a high incidence source of primary liver cancer worldwide. The fatality rate of liver cancer is still relatively high. Among the many treatment methods, liver resection is the first-line treatment of primary liver cancer. Although precision hepatectomy has achieved rapid development in recent years, the understanding of its efficacy is still not completely clear. This study aimed to analyze and compare the safety and effectiveness of precision hepatectomy and traditional hepatectomy in the treatment of primary liver cancer.

Methods: We performed a literature search of the CNKI, Wanfang, Weipu.com, PubMed, Cochrane Library, Web of Science databases for studies on precision liver resection (precision group) and traditional liver resection (traditional group) for the treatment of primary liver cancer. Data including the operation time, intraoperative blood loss, hospital stay, postoperative complications, liver function, and survival rate were analyzed using RevMan 5.3 software to compare the differences in the effects of the two surgical procedures.

Results: Ten articles were included in the study, involving a total of 1,969 patients, including 1,045 cases in the precision group and 924 cases in the traditional group. Meta-analysis results showed that compared with the traditional group, the precision group had a longer operation time [mean difference $(\mathrm{MD})=8.01$, $\mathrm{P}=0.004$ ], and total bilirubin (TBiL; $\mathrm{MD}=-2.78, \mathrm{P}=0.055)$ was similar. Meanwhile, the precision group exhibited advantages in terms of intraoperative blood loss $(\mathrm{MD}=-149.37, \mathrm{P}=0.000)$, hospital stay $(\mathrm{MD}=-5.59$, $\mathrm{P}=0.000$ ), postoperative liver function indexes [aspartate aminotransferase (AST; MD =-11.61, $\mathrm{P}=0.000$ ) and alanine aminotransferase (ALT; $M D=-18.53, \mathrm{P}=0.000)$ ], postoperative complication rate [relative risk $(\mathrm{RR})$ $=0.51, \mathrm{P}=0.000]$, and 1-year survival rate $(\mathrm{RR}=1.11, \mathrm{P}=0.000)$.

Discussion: The application of precision surgery in the treatment of primary liver cancer can be a safe and effective method. It can minimize intraoperative blood loss, mitigate surgical risk, reduce postoperative complications, improve patient prognosis and quality of life, and provide better short-term curative effect and patient benefits.

Keywords: Precise hepatectomy; primary liver cancer; traditional hepatectomy; meta-analysis

Submitted Oct 12, 2021. Accepted for publication Dec 06, 2021.

doi: 10.21037/jgo-21-735

View this article at: https://dx.doi.org/10.21037/jgo-21-735 


\section{Introduction}

Liver cancer is a malignant tumor with a high incidence worldwide. At present, surgical treatment is still an effective method for primary liver cancer. Since Chinese scholar Dong et al. (1) proposed precise hepatectomy as a new concept of liver surgery, it has aroused the attention of scholars worldwide. In recent years, with the comprehensive application of modern imaging technology and computerassisted systems, surgeons have applied the concept and technology of precise liver resection to perform liver surgery, in order to improve the accuracy of surgery (2-5). The core goal of surgical treatment is to achieve the complete resection of liver lesions. It also accurately assesses the maximum compensatory ability of the remaining liver postoperatively, and obtains the best therapeutic effect with minimal trauma $(6,7)$.

Surgical treatment of primary liver cancer has undergone various development stages, including wedge liver resection, regular liver resection, irregular local liver resection, and anatomical segment resection. In recent years, with the continuous development of related disciplines and technical equipment, precision and minimally invasive, and effective have become the development trend of liver surgery. Greater attention has been paid to precision liver resection, especially through emphasizing preoperative condition assessment, surgical planning, and perioperative treatment, with the aim of minimizing trauma, maximizing liver function protection, and providing the best rehabilitation effect $(2,8)$. Precision hepatectomy requires anatomical dissection of the intrahepatic blood vessels and bile duct system, as well as accurate and reliable treatment to protect the residual liver volume, reduce bleeding and blood transfusion, improve the curative effect, and provide greater benefit to patients. Therefore, it has become one of the main surgical treatments for primary liver cancer (9-12).

The primary aim of this study was to consult relevant literature on the clinical efficacy of precision hepatectomy and traditional hepatectomy for the treatment of primary hepatocellular carcinoma. Meta-analysis was used to analyze the operation time, intraoperative blood loss, postoperative hospital stay, postoperative liver function indicators, postoperative complications, and 1-year survival rate after surgery. Other short-term clinical effects were also analyzed, so as to comprehensively compare the effectiveness and safety of these two surgical methods. We present the following article in accordance with the PRISMA reporting checklist (available at https://dx.doi.org/10.21037/jgo-21-735).

\section{Methods}

\section{Search strategy}

We performed a literature search of the PubMed, Cochrane Library, Web of Science, CNKI, Wanfang, and Weipu databases. Articles published between January 1, 2009 and June 1, 2021 were searched using keywords. The search terms were as follows: precision hepatectomy, precision liver resection, anatomical liver resection, traditional liver resection, anatomical hepatectomy, primary liver cancer, hepatocellular carcinomas, liver cell carcinoma, and primary hepatocellular carcinoma. A combination of free words and subject terms was used as a search strategy, and reviews and references related to this research were also included.

\section{Inclusion criteria}

Articles published between January 1, 2009 and June 1, 2021 were eligible for inclusion in this meta-analysis. The inclusion criteria were as follows: (I) the postoperative pathology of all cases met the diagnostic criteria for primary liver cancer; (II) the intervention and treatment measures for primary liver cancer included precision liver resection and traditional liver resection; (III) the experimental design was a randomized controlled trial or a clinical controlled trial; (IV) observation indicators included at least operation time, intraoperative blood loss, average length of hospital stay, complication rate, postoperative 1 -year survival rate, as well as the postoperative aspartate transaminase (AST), alanine transaminase (ALT), and total bilirubin (TBiL) indicators; (V) liver function indicators were good, Child score as $\mathrm{A} / \mathrm{B}$, or $\mathrm{C}$ was converted to $\mathrm{B}$ after medical treatment; and (VI) cases involving no major underlying disease that could not tolerate surgery.

\section{Exclusion criteria}

The exclusion criteria were as follows: (I) literature that does not propose a controlled study, but is only a case report or a review; (II) articles with low scores, poor quality, little information, and no data involving observation indicators; (III) studies where the data could not be obtained by computer; and (IV) if the article involves duplicate data published by the same center or was published after 
expanding the sample size, the higher quality articles were selected.

\section{Paper screening and data extraction}

According to the predetermined inclusion and exclusion criteria, two professional researchers screened the literature. A third researcher participated in the decision in cases of disagreements between the two researchers. Data extraction was also based on the content of the literature, formulating a standardized table for independent data extraction, including basic information such as the name, author, and publication time of the included literature. Precision hepatectomy patients were included in the precision group, and traditional hepatectomy patients were included in the traditional group.

\section{Quality assessment}

The quality evaluation standards of the Cochrane Reviewers' Handbook were used to evaluate the quality of the included literature. The evaluation standards were as follows: (I) random allocation was utilized; (II) whether the allocation plan was informed; (III) whether blinding was used; (IV) whether the data results were lost; (V) whether the experimental results were selected for reporting; and (VI) whether there are other sources of bias. Different researchers evaluated the quality of the selected articles, exchanged different opinions, and sought third-party quality evaluation results.

\section{Statistical analysis}

RevMan version 5.3 software (Cochrane Collaboration, UK) was used for data analysis. Measurement data were expressed by the mean difference (MD) and 95\% confidence interval (CI), and count data were expressed by the relative risk (RR) and 95\% CI. A heterogeneity test was adopted for each outcome index of the included literature. If $\mathrm{P}>0.05$ and $\mathrm{I}^{2} \leq 50 \%$, the included studies were considered homogenous, and a fixed-effects model was used for meta-analysis; otherwise, if $\mathrm{P} \leq 0.05$ and $\mathrm{I}^{2}>50 \%$, the included studies were considered heterogeneous, and a random effects model was used for meta-analysis. $\mathrm{P}<0.05$ was considered to indicate a statistically significant difference in the outcome indicators included in the literature.

\section{Results}

\section{Search results and study characteristics}

In this study, we searched for articles published between January 1, 2009 and June 1, 2021 in the PubMed, Cochrane Library, Web of Science, CNKI, Wanfang, and Weipu databases. A total of 1,052 related articles were imported into the Note Express software (Beijing Aegean Software Company, China), including 318 Chinese articles and 734 English articles. According to the screening strategy and cased on the inclusion/exclusion criteria as well as a careful reading of the full texts, 10 high-quality articles were finally included. This included five Chinese articles and five English articles, involving a total of 1,969 cases, with 1,045 cases in the precision group and 924 cases in the traditional group. Figure 1 displays a flow chart of the established screening strategy. The basic characteristics of the 10 articles included in the study, including the author, publication year, and publication journal, are shown in Table 1 .

\section{Meta analysis results}

\section{Operating time}

Four of the included articles compared the operation time. The total number of included cases in the precision and traditional groups was 217 , respectively. The analysis results showed that $\mathrm{P}=0.589$ and $\mathrm{I}^{2}=0 \%$, indicating that there was homogeneity between the outcome indicators, so the fixed effects model was used for combined analysis. As shown in Figure 2, the combined effect size MD 8.01, 95\% CI: 2.60 13.42 , and the result of the comprehensive effect size test was $\mathrm{Z}=2.903, \mathrm{P}=0.004$. The meta-analysis showed that the difference in operation time between the two groups was statistically significant. The operation time of the precision group was longer than that of the traditional group.

\section{Intraoperative blood loss}

Six of the included articles compared the amount of intraoperative blood loss. The total number of included cases in the precision and traditional groups was 312 and 310 , respectively. The analysis results showed that $\mathrm{P}=0.000$ and $\mathrm{I}^{2}=96.5 \%$, indicating that there was heterogeneity between the result indicators, so the random effects model was used for combined analysis. As shown in Figure 3, the combined effect size MD $=-149.37,95 \% \mathrm{CI}:-186.12$ to -112.63 , and the combined effect size test result was 


\section{Identification of studies via databases and registers}

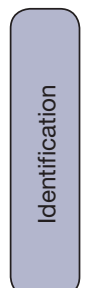

Records identified from the English database of PubMed, EMBASE, Web of Science, Cochrane Library, CNKI, Wanfang, Weipu: Databases $(n=1,100)$ Registers $(n=1)$
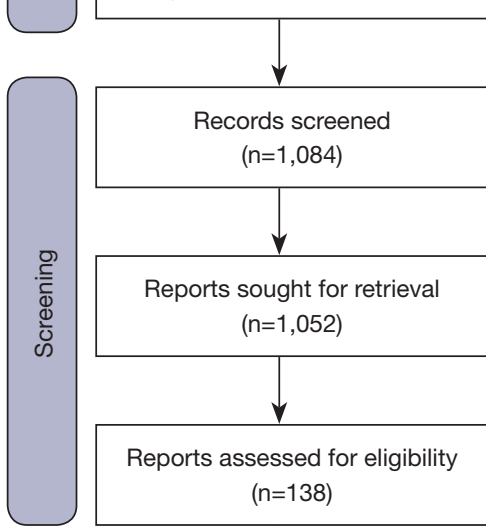

Reports sought for retrieval $(n=1,052)$

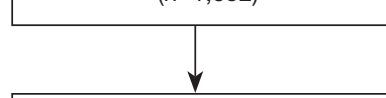

Reports assessed for eligibility $(n=138)$
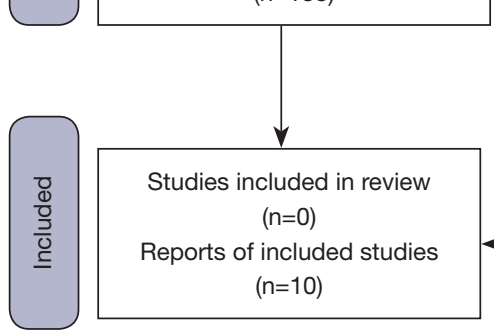

Records removed before screening: Duplicate records removed $(n=0)$ Records marked as ineligible by automation tools $(n=0)$

Records removed for other reasons $(n=17)$
Records excluded due to low quality $(n=32)$

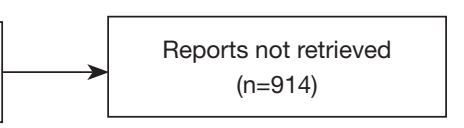

Reports excluded: Incomplete data $(n=35)$ Do not meet the criteria $(n=18)$ Non-randomized controlled trials $(n=49)$

Exclude ungraded liver function $(n=26)$

\section{Identification of studies via other methods}

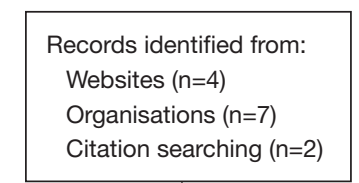

Records identified from:

sites $(n=4)$

Citation searching $(n=2)$
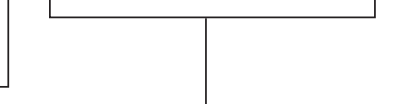

Figure 1 Research flow chart included in the meta-analysis.

Table 1 Basic characteristics of the included articles

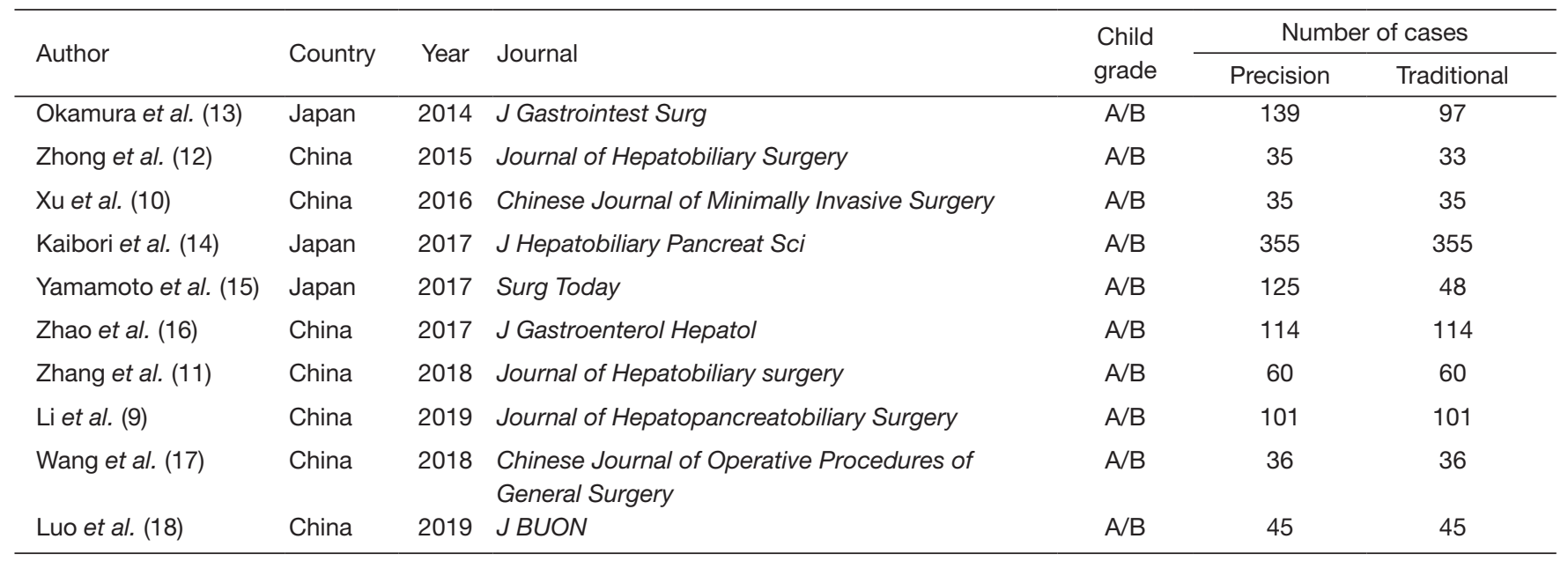




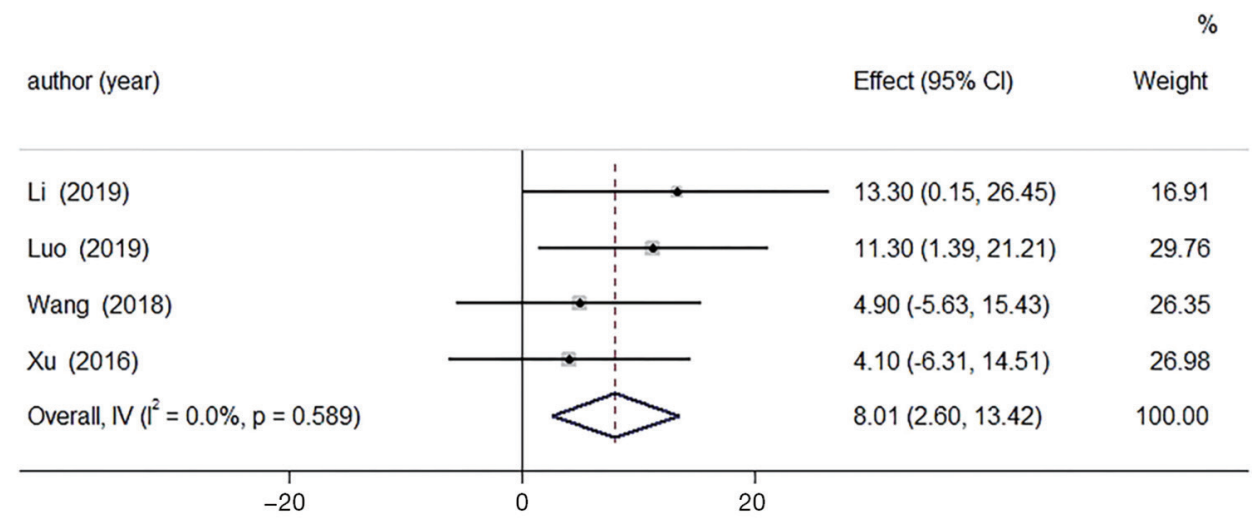

Figure 2 Forest plot of operating time using a fixed effects model. Comparison of operation time between precision group and traditional group. Statistical method: inverse variance of fixed effects model [mean difference (MD) and 95\% confidence interval (CI)].

\begin{tabular}{|c|c|c|c|}
\hline author (year) & & Effect $(95 \% \mathrm{Cl})$ & Weight \\
\hline$\longrightarrow$ & & $-204.40(-221.68,-187.12)$ & 16.77 \\
\hline Luo (2019) & $\longrightarrow$ & $-80.90(-96.39,-65.41)$ & 16.89 \\
\hline Wang (2018) & & $-172.70(-188.19,-157.21)$ & 16.89 \\
\hline Xu (2016) & & $-164.70(-184.13,-145.27)$ & 16.61 \\
\hline Zhang (2018) & & $-154.92(-183.54,-126.30)$ & 15.75 \\
\hline Zhong (2015) & $\longrightarrow$ & $-120.00(-132.06,-107.94)$ & 17.09 \\
\hline Overall, DL $\left(1^{2}=96.5 \%, p=0.000\right)$ & 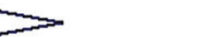 & $-149.37(-186.12,-112.63)$ & 100.00 \\
\hline $\begin{array}{c}1 \\
-200\end{array}$ & & & \\
\hline
\end{tabular}

NOTE: Weights are from random-effects model

Figure 3 Forest plot of intraoperative blood loss. Comparison of intraoperative blood loss between the precision group and the traditional group. Statistical method: inverse variance of the random effects model [mean difference (MD) and 95\% confidence interval (CI)].

$Z=-7.968, P=0.000$. The meta-analysis showed that the difference in intraoperative blood loss between the two groups was statistically significant, and the intraoperative blood loss in the precision group was less than that in the traditional group.

\section{Average length of hospital stay}

Six of the included articles compared the average length of hospital stay. The total number of included cases in the precision and traditional groups was 312 and 310 , respectively. The analysis results showed that $\mathrm{P}=0.000$ and $\mathrm{I}^{2}=95.8 \%$, indicating that there was heterogeneity between the result indicators, so the random effects model was used for combined analysis. As shown in Figure 4, the combined effect size MD $=-5.59,95 \%$ CI: -8.04 to -3.14 , and the combined effect size test result was $Z=-4.468, P=0.000$. The meta-analysis results showed that the difference in the average length of hospital stay between the two groups was statistically significant, and the average length of hospital stay in the precision group was shorter than that in the traditional group.

\section{Postoperative liver function}

Five of the included articles compared the levels of alanine aminotransferase (ALT). The total number of included cases in the precision and traditional groups was 211 and 209, respectively. The analysis results showed that $\mathrm{P}=0.000$ and $\mathrm{I}^{2}=98 \%$, indicating that there was heterogeneity between the outcome indicators, so the random effects model was used for combined analysis. As shown in Figure 5A, the combined 


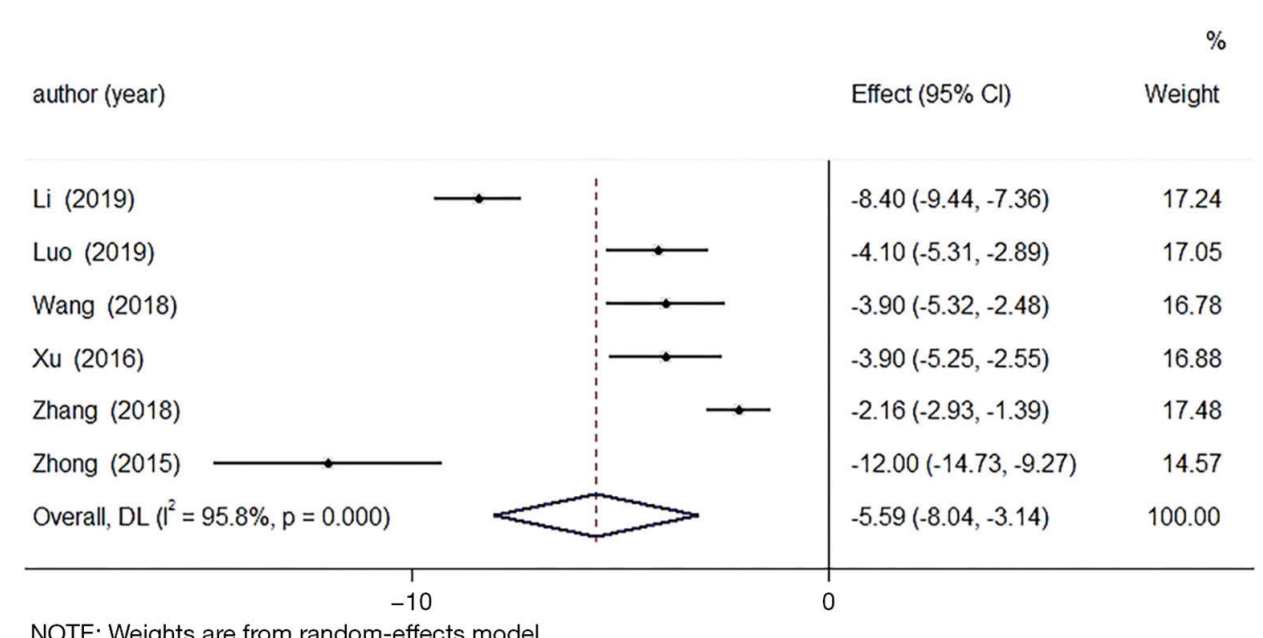

Figure 4 Forest plot of average length of hospital stay. Comparison of average length of hospital stay between the precision group and the traditional group. Statistical method: inverse variance of the random effects model [mean difference (MD) and 95\% confidence interval (CI)].

effect size $M D=-18.53,95 \%$ CI: -25.68 to -11.38 , and the combined effect size test result was $\mathrm{Z}=-50.08, \mathrm{P}=0.000$. The meta-analysis results showed that the difference in ALT levels between the two groups was statistically significant.

Five of the articles compared the levels of aspartate aminotransferase (AST). The total number of included cases in the precision and traditional groups was 211 and 209 , respectively. The analysis results $\left(\mathrm{P}=0.000, \mathrm{I}^{2}=97.4 \%\right)$ showed that there was heterogeneity between the outcome indicators, so the random effects model was used for combined analysis. As shown in Figure 5 B, the combined effect size $M D=-11.61,95 \%$ CI: -16.87 to -6.35 , and the combined effect size test result was $Z=-4.326, P=0.000$. The meta-analysis result showed that the difference in the levels of AST between the two groups was statistically significant.

Five of the included articles compared the levels of TBiL. The total number of included cases in the precision and traditional groups was 211 and 209, respectively. The analysis results $\left(\mathrm{P}=0.000, \mathrm{I}^{2}=97.1 \%\right)$ showed that there was heterogeneity between the outcome indicators, so the random effects model was used for combined analysis. As shown in Figure 5C, the combined effect size $\mathrm{MD}=-2.78$, 95\% CI: -5.62 to 0.06 , and the combined effect size test result was $Z=-1.916, P=0.055$. The meta-analysis results showed that the difference in the TBiL levels between the two groups was not statistically significant.

\section{Complication rate}

Five of the included articles compared the complication rate. The total number of included cases in the precision and traditional groups was 366 and 289, respectively. The analysis results $\left(\mathrm{P}=0.304, \mathrm{I}^{2}=17.4 \%\right)$ indicated that there was homogeneity between the outcome indicators, so the fixed effects model was used for combined analysis. As shown in Figure 6, the combined effect size RR $=0.51,95 \%$ CI: $0.36-0.73$, and the combined effect size test result was $\mathrm{Z}=-3.687, \mathrm{P}=0.000$. The meta-analysis results showed that the incidence of complications between the two groups was statistically significant, and the incidence of postoperative complications was lower in the precision group.

\section{One-year survival rate postoperatively}

Six of the included articles that compared the 1-year survival rate after surgery. The total number of included cases in the precision and traditional groups was 894 and 775 , respectively. The analysis results $\left(\mathrm{P}=0.311, \mathrm{I}^{2}=16.0 \%\right)$ showed that there was homogeneity between the outcome indicators, so the fixed-effects model was used for combined analysis. As shown in Figure 7, the combined effect size RR $=1.11,95 \%$ CI: $1.05-1.17$, and the combined effect size test result was $Z=3.610, P=0.000$. The meta-analysis results showed that the difference in the survival rate of the two groups was statistically significant, and the 1-year survival rate of the precision group was higher.

\section{Publication bias}

A funnel chart was used to examine the publication bias of 


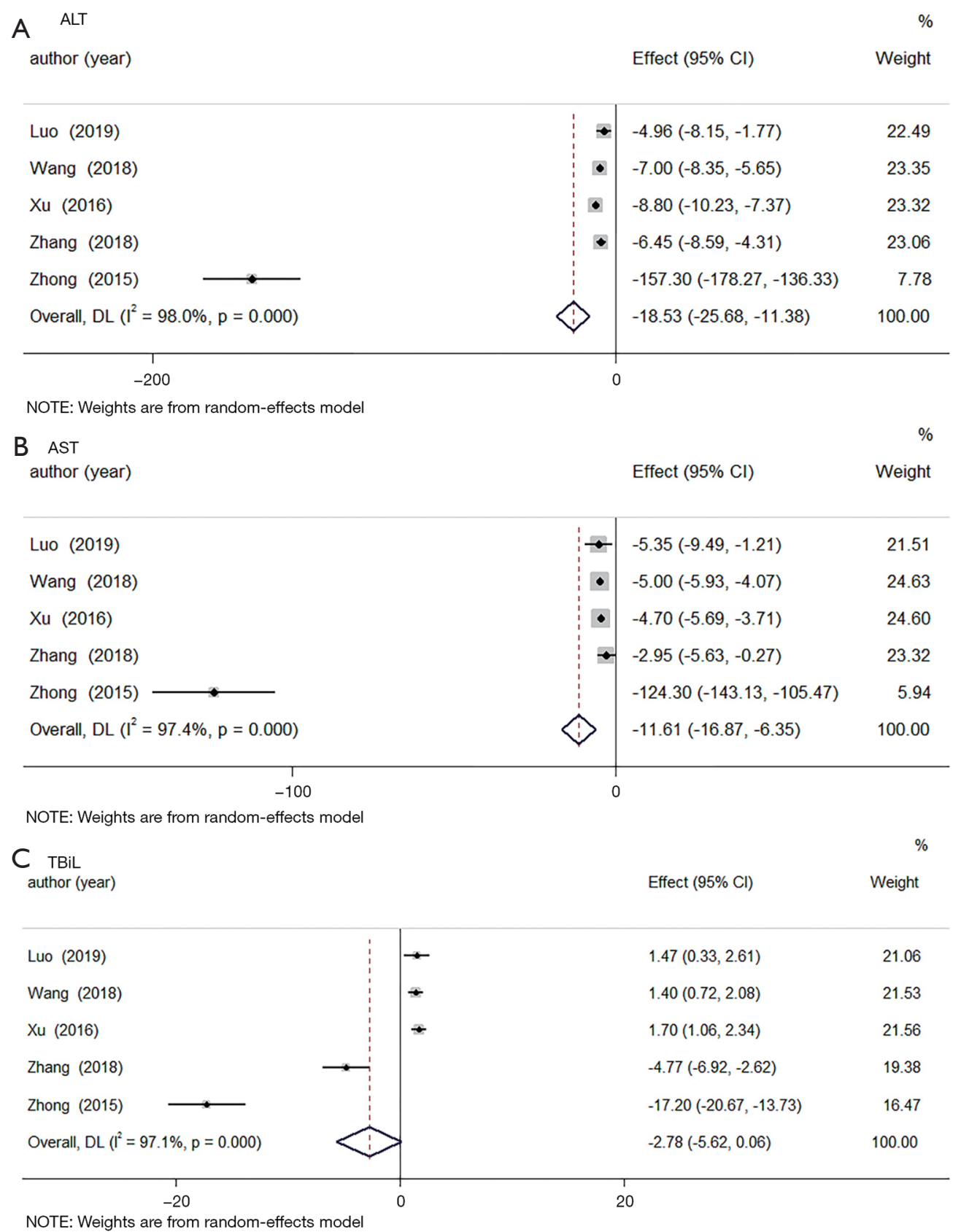

Figure 5 Forest plot of postoperative liver function. Comparison of postoperative liver function [alanine aminotransferase (ALT), aspartate aminotransferase (AST) and total bilirubin (TBiL)] between the precision group and the traditional group. Statistical method: inverse variance of the random effects model [mean difference (MD) and 95\% confidence interval (CI)]. 


\begin{tabular}{|c|c|c|}
\hline author (year) & $\begin{array}{l}\text { Risk Ratio } \\
(95 \% \mathrm{Cl})\end{array}$ & $\begin{array}{r}\% \\
\text { Weight }\end{array}$ \\
\hline Li (2019) & $0.46(0.25,0.87)$ & 34.16 \\
\hline Luo (2019) & $0.44(0.20,0.96)$ & 21.02 \\
\hline Xu (2016) & $0.25(0.06,1.09)$ & 10.51 \\
\hline Yamamoto (2017) & $1.10(0.50,2.43)$ & 13.29 \\
\hline Zhang (2018) & $0.44(0.19,0.99)$ & 21.02 \\
\hline Overall, $M H\left(l^{2}=17.4 \%, p=0.304\right)$ & $0.51(0.36,0.73)$ & 100.00 \\
\hline 0.0625 & & \\
\hline
\end{tabular}

NOTE: Weights are from Mantel-Haenszel model

Figure 6 Forest plot of complication rate. activity ability. Comparison of complication rate between the precision group and the traditional group. Statistical method: Mantel-Haenszel of fixed effects model [relative risk (RR) and 95\% confidence interval (CI)].

\begin{tabular}{|c|c|c|c|c|}
\hline author (year) & & & $\begin{array}{l}\text { Risk Ratio } \\
(95 \% \mathrm{Cl})\end{array}$ & $\begin{array}{r}\% \\
\text { Weight }\end{array}$ \\
\hline Kaibori (2017) & $\rightarrow$ & & $1.09(1.01,1.17)$ & 46.92 \\
\hline Li (2019) & & & $1.20(0.94,1.54)$ & 9.10 \\
\hline Okamura (2014) & & & $1.15(0.99,1.34)$ & 13.92 \\
\hline Yamamoto (2017) & 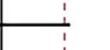 & & $0.92(0.75,1.12)$ & 9.04 \\
\hline Zhang (2018) & & & $1.31(1.02,1.70)$ & 6.08 \\
\hline Zhao (2017) & & & $1.12(0.98,1.27)$ & 14.94 \\
\hline Overall, $\mathrm{MH}\left(\mathrm{l}^{2}=16.0 \%, \mathrm{p}=0.311\right)$ & & & $1.11(1.05,1.17)$ & 100.00 \\
\hline${ }_{0.6666667}^{1}$ & 1 & 1.5 & & \\
\hline
\end{tabular}

Figure 7 Forest plot of 1-year survival rate. Comparison of 1-year survival rate between the precision group and the traditional group. Statistical method: Mantel-Haenszel of fixed effects model [relative risk (RR) and 95\% confidence interval (CI)].

the operating time and liver function. Both funnel charts showed asymmetry, indicating that there may be publication bias, as shown in Figure 8.

\section{Risk of bias}

Among the eligible studies, all articles described that random sequence generation is low risk. Four studies had a low risk of allocation concealment bias $(9,12,15,17)$, and all trials had a higher risk of bias for blinding participants and researchers. Furthermore, all trials described the risk of bias in blinded results assessment [low risk $=5(9,13,14,16,18)$, high risk $=5(10-12,15,17)]$. Also, all trials had a low risk of bias for incomplete outcome data. For the selective reporting domains, all studies were judged to have a low risk of bias.

\section{Discussion}

The concept of precision surgery was applied to the concept and technology of liver resection, including the entire treatment process. According to the preoperative assessment 

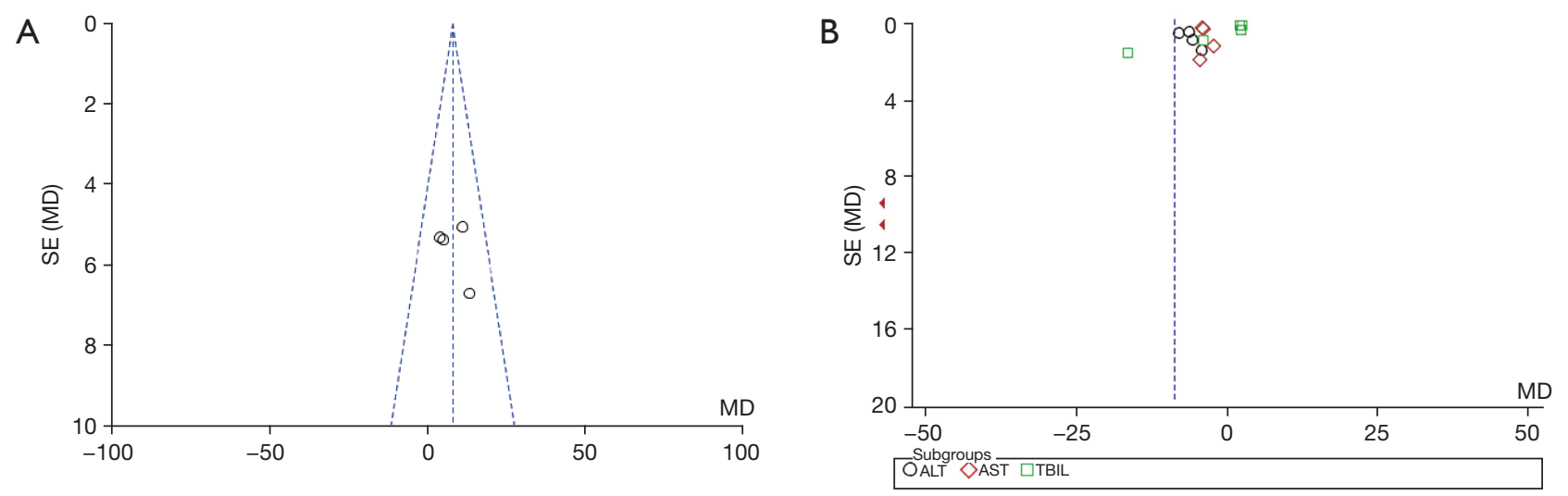

Figure 8 Funnel plot analysis of possible publication bias in subgroups. Operating time (A) and liver function (B) funnel chart. MD, mean difference; SE, standard error of the mean.

of different cases (1), various surgical strategies have been developed to enable the patient maximize benefits. In traditional hepatectomy, surgeons evaluate the lesion based on imaging data and clinical experience. It is difficult to fully grasp the anatomical relationship between the lesion and the vessel, as well as the amount of pre-resected liver and the resection boundary, and it is impossible to develop the optimal surgical plan. Therefore, traditional hepatectomy may increase the possibility of vascular and bile duct damage to patients, and increase the incidence of surgical complications in patients $(6,19,20)$. Precise liver resection is a surgical plan based on precise preoperative assessments for different cases, and is combined with new technologies $(2,5)$. It does not specifically refer to a certain advanced surgical technique, but rather aims to maximize patient benefits under the requirements of high precision and efficiency. The principle of benefit refers to the implementation of individualized and precise treatment for patients $(1,3,21)$.

The results of this study showed that the outcome indicators of the precision and traditional groups were statistically significant. Among them, the research revealed that the precision group had a longer operation time than the traditional group, and was superior to the traditional group in terms of the other indicators. Through careful reading of the included literature, the reasons for the above results are analyzed as follows. Precise liver resection emphasizes accurate preoperative assessment of the patient's liver function, coupled with fine surgical operations, as well as the use of three-dimensional imaging technology to increase the preoperative preparation time $(8,21)$. Given the implementation of precise liver parenchymal anatomy, the application of new instruments, and the measurement of residual liver volume, as well as the careful separation of the expected resection liver boundary, such a delicate operation further prolongs the operation time, thereby reducing intraoperative blood loss and minimizing the surgical risks. Liver cancer patients often have liver dysfunction, so postoperative residual liver volume is closely related to postoperative liver function (22-24). Smaller residual liver volume indicate a higher probability of liver insufficiency after surgery $(10,17)$. Due to this and the difference in the size and location of each patient's tumor, precise hepatectomy is required. Preoperatively, individualized principles are used for different patients to calculate the residual liver volume and fully evaluate the residual liver function, which can better preserve the residual liver.

Preserving the residual liver volume can improve the physiological function of the liver after surgery. Vauthey et al. (25) also reported that the amount of residual liver is critical and is closely related to severe postoperative complications; residual liver volumes below $25 \%$ significantly increase the incidence of complications. Du et al. (26) also confirmed that the volume of residual liver is closely related to the occurrence of liver failure after surgery, which is an important factor. Based on the large number of hepatitis B patients worldwide, the number of hepatitis B cirrhosis patients is also considerable. Clearly, calculating the amount of residual liver and evaluating residual liver function is particularly important. Following the concept of precision liver resection, the precision group's postoperative liver function indicators and complication rate exhibited advantages $(9,22)$. Precision liver resection uses new technologies, such as three-dimensional imaging and 
intraoperative ultrasound, to accurately locate the tumor margins, and uses delicate surgical techniques to completely resect lesions. Precision liver resection aims to maximize the protection of the functional liver parenchyma under the premise of ensuring the complete removal of the target lesions, thus achieving the maximum surgical benefit with the smallest surgical invasion, improving the 1-year survival rate of patients after surgery, and promoting the early and rapid complete recovery of patients.

This study has some limitations that should be noted. Firstly, some of the included literature may be due to the characteristics of the patient's own reasons, and it is necessary to choose an appropriate surgical plan based on the condition. Allocation cannot be completely randomized, nor can it be blinded. The quality evaluation of the literature also has a certain impact; although some patients can tolerate surgery, data regarding whether the patients also had other underlying diseases was not described in some literature. The number of literature included in this study is not enough, not representative, and only analyzed the outcome indicators of short-term efficacy. Therefore, we look forward to higher-quality long-term follow-up research results to evaluate the application of precision liver resection.

\section{Conclusions}

In summary, applying the concept of precision surgery to the treatment of primary liver cancer is a safe and effective method. Although the operation time is longer, it can reduce blood loss during surgery and with more residual liver volume, liver function can be effectively protected. At the same time, it can reduce postoperative complications and improve the 1-year survival rate of patients after surgery. Compared with traditional hepatectomy, precision liver resection has a superior curative effect.

\section{Acknowledgments}

Funding: None.

\section{Footnote}

Reporting Checklist: The authors have completed the PRISMA reporting checklist (available at https://dx.doi. org/10.21037/jgo-21-735).

Conflicts of Interest: All authors have completed the ICMJE uniform disclosure form (available at https://dx.doi. org/10.21037/jgo-21-735). The authors have no conflicts of interest to declare.

Ethical Statement: The authors are accountable for all aspects of the work in ensuring that questions related to the accuracy or integrity of any part of the work are appropriately investigated and resolved.

Open Access Statement: This is an Open Access article distributed in accordance with the Creative Commons Attribution-NonCommercial-NoDerivs 4.0 International License (CC BY-NC-ND 4.0), which permits the noncommercial replication and distribution of the article with the strict proviso that no changes or edits are made and the original work is properly cited (including links to both the formal publication through the relevant DOI and the license). See: https://creativecommons.org/licenses/by-nc-nd/4.0/.

\section{References}

1. Dong JH, Yang SZ, Duan WD, et al. Clinical application of precise liver resection techniques in patients with complicated liver space-occupying lesions. Zhonghua Wai Ke Za Zhi 2009;47:1610-5.

2. Lv A, Li Y, Qian HG, et al. Precise Navigation of the Surgical Plane with Intraoperative Real-time Virtual Sonography and 3D Simulation in Liver Resection. J Gastrointest Surg 2018;22:1814-8.

3. Su L, Zhou XJ, Dong Q, et al. Application value of computer assisted surgery system in precision surgeries for pediatric complex liver tumors. Int J Clin Exp Med 2015;8:18406-12.

4. Banchini F, Luzietti E, Cecconi S, et al. Achieving precision surgery in laparoscopic liver resection with the aid of preoperative three-dimensional reconstruction: A case report. Int J Surg Case Rep 2021;81:105792.

5. Xu Y, Chen M, Meng X, et al. Laparoscopic anatomical liver resection guided by real-time indocyanine green fluorescence imaging: experience and lessons learned from the initial series in a single center. Surg Endosc 2020;34:4683-91.

6. Ochiai T, Ikoma H, Inoue K, et al. Intraoperative realtime cholangiography and C-tube drainage in donor hepatectomy reduce biliary tract complications. J Gastrointest Surg 2011;15:2159-64.

7. Zhang J, Xu Y, Yang H, et al. Application of associating liver partition and portal vein ligation for staged 
hepatectomy for hepatocellular carcinoma related to hepatitis B virus: comparison with traditional one-stage right hepatectomy. Transl Cancer Res 2020;9:5371-9.

8. Dong J, Yang S, Zeng J, et al. Precision in liver surgery. Semin Liver Dis 2013;33:189-203.

9. Li L, Wang J, Luo M, et al. A comparative analysis of curative effect between precise hepatectomy and traditional hepatectomy for primary hepatocellular carcinoma. Journal of Hepatopancreatobiliary Surgery 2019;31:404-7.

10. Xu J, Wen Y, Li J, et al. Clinical Application Value of Laparoscopic Precise Liver Resection. Chinese Journal of Minimally Invasive Surgery 2016;16:590-3.

11. Zhang L, Zhang W, Wang G, et al. Clinical analysis of different hepatectomy under laparoscope in the treatment of primary liver cancer. Journal of Hepatobiliary Surgery 2018;26:119-22.

12. Zhong T, Lin J, He Q, et al. Clinical efficacy analysis of precise liver resection in the treatment of primary liver cancer. Journal of Hepatobiliary Surgery 2015;23:253-5.

13. Okamura Y, Ito T, Sugiura T, et al. Anatomic versus nonanatomic hepatectomy for a solitary hepatocellular carcinoma: a case-controlled study with propensity score matching. J Gastrointest Surg 2014;18:1994-2002.

14. Kaibori M, Kon M, Kitawaki T, et al. Comparison of anatomic and non-anatomic hepatic resection for hepatocellular carcinoma. J Hepatobiliary Pancreat Sci 2017;24:616-26.

15. Yamamoto T, Yagi S, Uryuhara K, et al. Clinical factors that affect the outcomes after anatomical versus nonanatomical resection for hepatocellular carcinoma. Surg Today 2017;47:193-201.

16. Zhao H, Chen C, Gu S, et al. Anatomical versus nonanatomical resection for solitary hepatocellular carcinoma without macroscopic vascular invasion: A propensity score matching analysis. J Gastroenterol Hepatol 2017;32:870-8.

17. Wang X, Zhang Y. Clinical outcome of laparoscopic precise liver resection in the treatment of primary liver

Cite this article as: Niu X, Liu J, Feng Z, Zhang T, Su T, Han W. Short-term efficacy of precise hepatectomy and traditional hepatectomy for primary liver cancer: a systematic review and meta-analysis. J Gastrointest Oncol 2021;12(6):3022-3032. doi: 10.21037/jgo-21-735 cancer. Chinese Journal of Operative Procedures of General Surgery (Electronic Version) 2018;12:379-81.

18. Luo F, Zhou J, Yan W. A comparative study of laparoscopic precise hepatectomy with conventional open hepatectomy in the treatment of primary hepatocellular cancer. J BUON 2019;24:1943-9.

19. Aoki T, Murakami M, Koizumi T, et al. Preoperative Tattooing for Precise and Expedient Localization of Landmark in Laparoscopic Liver Resection. J Am Coll Surg 2015;221:e97-e101.

20. Chanwat R. Useful maneuvers for precise laparoscopic liver resection. Asian J Endosc Surg 2018;11:93-103.

21. Chen XP, Zhang WD, Wang D, et al. Image classification of liver cancer surrounding right hepatic pedicle and its guide to precise liver resection. Int J Clin Exp Med 2015;8:11093-100.

22. Ni ZK, Lin D, Wang ZQ, et al. Precision Liver Resection: Three-Dimensional Reconstruction Combined with Fluorescence Laparoscopic Imaging. Surg Innov 2021;28:71-8.

23. Qian NS, Liao YH, Cai SW, et al. Comprehensive application of modern technologies in precise liver resection. Hepatobiliary Pancreat Dis Int 2013;12:244-50.

24. Tomimaru Y, Eguchi H, Marubashi S, et al. Equivalent outcomes after anatomical and non-anatomical resection of small hepatocellular carcinoma in patients with preserved liver function. Dig Dis Sci 2012;57:1942-8.

25. Vauthey JN, Chaoui A, Do KA, et al. Standardized measurement of the future liver remnant prior to extended liver resection: methodology and clinical associations. Surgery 2000;127:512-9.

26. Du ZG, Li B, Wei YG, et al. A new scoring system for assessment of liver function after successful hepatectomy in patients with hepatocellular carcinoma. Hepatobiliary Pancreat Dis Int 2011;10:265-9.

(English Language Editor: A. Kassem) 